\title{
Total Synthesis of (-)- and (+)-Membrenone C
}

\author{
James A. Marshall and Keith C. Ellis \\ Department of Chemistry, University of Virginia \\ Charlottesville, VA 22904 \\ jam5x@virginia.edu
}

Supporting Information

Experimental Prodedures for all compounds..............................pp S1-S18

Selected ${ }^{1} \mathrm{H}$ and ${ }^{13} \mathrm{C}$ NMR spectra............................................pp S19-S38 


\title{
Total Synthesis of (-)- and (+)-Membrenone C
}

\author{
James A. Marshall and Keith C. Ellis \\ Department of Chemistry, University of Virginia \\ Charlottesville, VA 22904 \\ jam5x@virginia.edu
}

\section{Supporting Information}

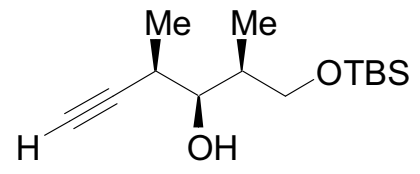

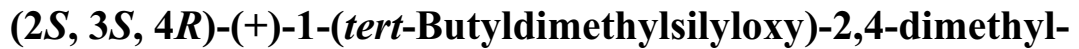

5-hexyn-3-ol (3). To a solution of $(P)$-allenylstannane $(P)$-1

$(0.733 \mathrm{~g}, 2.14 \mathrm{mmol})$ and (2S)-3-(tert-butyldimethylsilyloxy)-

2-methylpropanal $2(0.280 \mathrm{~g}, 1.38 \mathrm{mmol})$ in $\mathrm{CH}_{2} \mathrm{Cl}_{2}(14 \mathrm{~mL})$ at $-78{ }^{\circ} \mathrm{C}$ was added $\mathrm{BF}_{3} \cdot \mathrm{OEt}_{2}(0.50 \mathrm{~mL}, 4.22 \mathrm{mmol})$ dropwise over $5 \mathrm{~min}$. After $1 \mathrm{~h}$ the reaction was quenched at $-78{ }^{\circ} \mathrm{C}$ by addition of $10 \mathrm{~mL}$ of $\mathrm{MeOH} / \mathrm{NaHCO}_{3}$ (sat.) $(5: 1, \mathrm{v} / \mathrm{v})$ and warmed to room temperature. The layers were separated and the aqueous layer was extracted with ether. The combined organic extracts were washed with brine, dried over $\mathrm{MgSO}_{4}$, filtered, and concentrated under reduced pressure. Purification by gradient elution chromatography on silica gel (0\% -5\% EtOAc/hexanes) afforded $0.289 \mathrm{~g} \mathrm{(82 \% )}$ of a colorless oil. $\mathrm{R}_{\mathrm{f}}=0.80$ (25\% EtOAc/hexanes); $[\alpha]_{\mathrm{D}}^{20}=+1.61$ (c 2.1, $\left.\mathrm{CHCl}_{3}\right)$; IR (film) 3490, 3310, 2965, $2365 \mathrm{~cm}^{-1}$; ${ }^{1} \mathrm{H}$ NMR (300 MHz, $\left.\mathrm{CDCl}_{3}\right)-3.89(1 \mathrm{H}, \mathrm{dd}, J=2.7,9.6$ $\mathrm{Hz}), 3.72(2 \mathrm{H}, \mathrm{dd}, J=2.7,9.9 \mathrm{~Hz}), 3.51(1 \mathrm{H}, \mathrm{br} \mathrm{s}), 2.52(1 \mathrm{H}, \mathrm{qt}, J=2.7,6.9 \mathrm{~Hz}), 2.16$ $(1 \mathrm{H}, \mathrm{m}), 2.06(1 \mathrm{H}, \mathrm{d}, J=2.4 \mathrm{~Hz}), 1.31(3 \mathrm{H}, \mathrm{d}, J=6.6 \mathrm{~Hz}), 1.01(3 \mathrm{H}, \mathrm{d}, J=7.2 \mathrm{~Hz}), 0.90$ $(9 \mathrm{H}, \mathrm{s}), 0.09(6 \mathrm{H}, \mathrm{d}, J=0.9 \mathrm{~Hz}) ;{ }^{13} \mathrm{C} \mathrm{NMR}\left(75.5 \mathrm{MHz}, \mathrm{CDCl}_{3}\right)$ 86.35, 78.26, 69.88, $69.40,36.26,30.20,25.83,18.14,17.66,9.36,-5.71$.

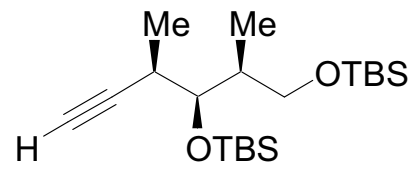

$(2 S, 3 S, 4 R)-(+)-1,3-B i s(t e r t-b u t y l d i m e t h y l s i l y l o x y)-2,4-$ dimethyl-5-hexyn-3-ol (4). To a solution of alcohol 3 (4.125 
g, $16.1 \mathrm{mmol})$ in $\mathrm{CH}_{2} \mathrm{Cl}_{2}(161 \mathrm{~mL})$ at $0{ }^{\circ} \mathrm{C}$ was added 2,6-lutidine $(3.75 \mathrm{~mL}, 32.2 \mathrm{mmol})$ and TBSOTf $(5.6 \mathrm{~mL}, 24.2 \mathrm{mmol})$. The mixture was allowed to warm to room temperature and stirred for $1.5 \mathrm{~h}$. The reaction was quenched with $\mathrm{H}_{2} \mathrm{O}(50 \mathrm{~mL})$, and the aqueous layer was extracted with $\mathrm{CH}_{2} \mathrm{Cl}_{2}$, dried over $\mathrm{MgSO}_{4}$, filtered, and concentrated under reduced pressure. Purification by chromatography on silica gel (1\% EtOAc/hexanes) afforded $4.04 \mathrm{~g}(87 \%)$ of a colorless oil. $\mathrm{R}_{\mathrm{f}}=0.95(25 \%$ EtOAc/hexanes); $[\alpha]^{20}{ }_{\mathrm{D}}=+7.7$ (c 1.0, $\mathrm{CHCl}_{3}$ ); IR (film) 3325, 2943, $2864 \mathrm{~cm}^{-1} ;{ }^{1} \mathrm{H}$ $\operatorname{NMR}\left(300 \mathrm{MHz}, \mathrm{CDCl}_{3}\right) 3.76(1 \mathrm{H}, \mathrm{dd}, J=1.8,7.8 \mathrm{~Hz}), 3.43(2 \mathrm{H}, \mathrm{dq}, J=1.5,8.4 \mathrm{~Hz})$, $2.59(1 \mathrm{H}, \mathrm{qd}, J=2.4,6.9 \mathrm{~Hz}), 2.12(1 \mathrm{H}, \mathrm{m}), 2.05(1 \mathrm{H}, \mathrm{d}, J=2.7 \mathrm{~Hz}), 1.20(3 \mathrm{H}, \mathrm{d}, J=6.9$ $\mathrm{Hz}), 0.90(18 \mathrm{H}, \mathrm{s}), 0.86(3 \mathrm{H}, \mathrm{d}, J=6.9 \mathrm{~Hz}), 0.08(6 \mathrm{H}, \mathrm{s}), 0.05(6 \mathrm{H}, \mathrm{d}, J=1.8 \mathrm{~Hz}) ;{ }^{13} \mathrm{C}$ NMR (75.5 MHz, $\left.\mathrm{CDCl}_{3}\right)$ 74.30, 69.80, 65.60, 39.34, 30.97, 26.11, 25.90, 18.42, 18.18, 10.11, -3.84, -4.16, -5.25. Anal. Calcd for $\mathrm{C}_{20} \mathrm{H}_{42} \mathrm{O}_{2} \mathrm{Si}_{2}$ : C, 64.80; H, 11.42. Found: $\mathrm{C}$, $64.53 ; \mathrm{H}, 11.63$.

\section{$(2 S, 3 S, 4 R)-(+)-3-(t e r t-B u t y l d i m e t h y l s i l y l o x y)-2,4-d i m e t h y l-5-$}

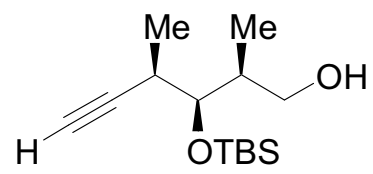

hexyn-1-ol (5). Bis-silyl ether 4 (0.108 g, $0.29 \mathrm{mmol})$ was taken up in $95 \% \mathrm{EtOH}(2.7 \mathrm{~mL})$ and PPTS $(8 \mathrm{mg}, 0.03 \mathrm{mmol})$ was added at room temperature. After the reaction was judged complete by TLC (approximately 48 to $60 \mathrm{~h}$ ), brine $(5 \mathrm{~mL})$ was added and the mixture was extracted with ether, dried over $\mathrm{MgSO}_{4}$, filtered, and concentrated under reduced pressure. Purification by chromatography on silica gel (10\% EtOAc/hexanes) afforded $0.069 \mathrm{~g}(92 \%)$ of a yellow oil. $\mathrm{R}_{\mathrm{f}}=0.72\left(25 \%\right.$ EtOAc/hexanes); $[\alpha]^{20}{ }_{\mathrm{D}}=+6.8\left(c 0.99, \mathrm{CHCl}_{3}\right)$; IR (film) 3318, 2924, $2484 \mathrm{~cm}^{-1}$; ${ }^{1} \mathrm{H}$ NMR (300 MHz, $\left.\mathrm{CDCl}_{3}\right) 3.76(1 \mathrm{H}, \mathrm{dd}, J=2.1,7.5 \mathrm{~Hz}), 3.59$ $(1 \mathrm{H}, \mathrm{dd}, J=8.4,10.5 \mathrm{~Hz}), 3.51(1 \mathrm{H}, \mathrm{dd}, J=6.0,10.5 \mathrm{~Hz}), 2.63(1 \mathrm{H}, \mathrm{m}), 2.19(1 \mathrm{H}, \mathrm{m})$, $2.08(1 \mathrm{H}, \mathrm{d}, J=2.4 \mathrm{~Hz}), 1.66(1 \mathrm{H}, \mathrm{br} \mathrm{s}), 1.22(3 \mathrm{H}, \mathrm{d}, J=6.9 \mathrm{~Hz}), 0.93(3 \mathrm{H}, \mathrm{d}, J=6.9$ $\mathrm{Hz}), 0.91(9 \mathrm{H}, \mathrm{s}), 0.10(6 \mathrm{H}, \mathrm{s}) ;{ }^{13} \mathrm{C} \mathrm{NMR}\left(75.5 \mathrm{MHz}, \mathrm{CDCl}_{3}\right)$ 87.14, 75.48, 70.20, 65.76, 39.90, 30.38, 26.03, 18.35, 10.89, -3.84, -4.20. Anal. Calcd for $\mathrm{C}_{14} \mathrm{H}_{28} \mathrm{O}_{2} \mathrm{Si}$ : C, 65.57; H, 11.00. Found: C, 65.34; H, 11.07. 


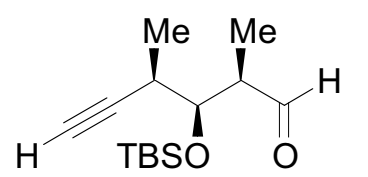

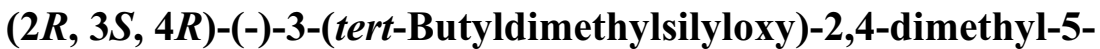
hexynal (6). To a stirring solution of oxalyl chloride $(0.25 \mathrm{~mL}, 2.9$ $\mathrm{mmol})$ in $\mathrm{CH}_{2} \mathrm{Cl}_{2}(20 \mathrm{~mL})$ at $-78^{\circ} \mathrm{C}$ was added DMSO $(0.40 \mathrm{~mL}$, $5.6 \mathrm{mmol})$. After $15 \mathrm{~min}$, alcohol $5(0.484 \mathrm{~g}, 1.90 \mathrm{mmol})$ in $\mathrm{CH}_{2} \mathrm{Cl}_{2}(1 \mathrm{~mL})$ was added. After $15 \mathrm{~min}$, triethylamine $(1.20 \mathrm{~mL}, 8.6 \mathrm{mmol})$ was added over 30 minutes by means of a syringe pump. The reaction mixture was stirred at $-78^{\circ} \mathrm{C}$ for $3 \mathrm{~h}$, then warmed to -40 ${ }^{\circ} \mathrm{C}$. After $10 \mathrm{~min}$, the reaction was quenched with saturated $\mathrm{NaHCO}_{3}(20 \mathrm{~mL})$, and warmed to room temperature. The layers were separated and the aqueous layer was washed two times with $\mathrm{CH}_{2} \mathrm{Cl}_{2}$. The combined organic layers were washed with brine, dried over $\mathrm{MgSO}_{4}$, filtered, and concentrated under reduced pressure. The crude oil was triturated with $10 \%$ EtOAc/hexanes, filtered, and concentrated to afford $0.472 \mathrm{~g}(98 \%)$ of light yellow oil which was used without further purification. $\mathrm{R}_{\mathrm{f}}=0.80(25 \%$ EtOAc/hexanes); $[\alpha]^{20}{ }_{\mathrm{D}}=-35.2$ (c 1.3 $\mathrm{CHCl}_{3}$ ); IR (film) 3327, 2968, 2881, $1736 \mathrm{~cm}^{-1}$; ${ }^{1} \mathrm{H}$ NMR $\left(300 \mathrm{MHz}, \mathrm{CDCl}_{3}\right) 9.74(1 \mathrm{H}, \mathrm{s}), 4.17(1 \mathrm{H}, \mathrm{dd}, J=2.1,8.1 \mathrm{~Hz}), 2.91(1 \mathrm{H}, \mathrm{dq}, J$ $=1.8,6.9 \mathrm{~Hz}), 2.61(1 \mathrm{H}, \mathrm{m}), 1.25(3 \mathrm{H}, \mathrm{d}, J=7.2 \mathrm{~Hz}), 1.17(3 \mathrm{H}, \mathrm{d}, J=6.9 \mathrm{~Hz}), 0.90(1 \mathrm{H}$, d, $J=1.2 \mathrm{~Hz}), 0.87(9 \mathrm{H}, \mathrm{s}), 0.10(3 \mathrm{H}, \mathrm{s}),-0.01(3 \mathrm{H}, \mathrm{s}) ;{ }^{13} \mathrm{C} \mathrm{NMR}\left(75.5 \mathrm{MHz}, \mathrm{CDCl}_{3}\right)$ $86.08,74.10,71.02,51.01,31.20,25.90,18.24,17.94,7.00,-4.13,-4.12$.

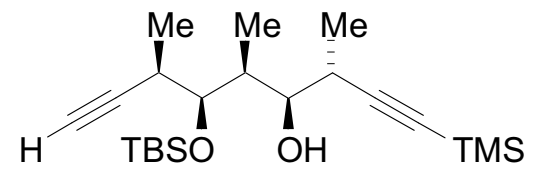

\section{$(3 R, 4 R, 5 R, 6 S, 7 R)-(+)-6-(t e r t-$}

\section{Butyldimethylsilyloxy)-3,5,7-trimethyl-1-}

trimethylsilylnonan-1,8-diyn-4-ol (8). To a stirring solution of aldehyde 6 (2.262 $\mathrm{g}, 8.90 \mathrm{mmol})$ in THF/HMPA $(3: 1,45 \mathrm{~mL})$ was added $\mathrm{Pd}(\mathrm{OAc})_{2}(0.121 \mathrm{~g}, 0.54 \mathrm{mmol})$. The reaction was cooled to $0{ }^{\circ} \mathrm{C}$ and $\mathrm{PPh}_{3}(0.142 \mathrm{~g}$, $0.54 \mathrm{mmol}$ ) was added. Upon complete dissolution of the $\mathrm{PPh}_{3}$, pulverized $\mathrm{InI}$ ( $3.268 \mathrm{~g}$, $13.5 \mathrm{mmol})$ was added, followed by the mesylate of $(S)$-4-TMS-3-butyn-2-ol (2.83 g, $12.8 \mathrm{mmol}$ ). After stirring at $0{ }^{\circ} \mathrm{C}$ for $2 \mathrm{~h}$, the reaction was quenched by pouring into a stirring solution of $\mathrm{NH}_{4} \mathrm{Cl}(100 \mathrm{~mL}), 10 \% \mathrm{HCl}(50 \mathrm{~mL})$, and $\mathrm{Et}_{2} \mathrm{O}(100 \mathrm{~mL})$. The layers were separated and the aqueous layer was washed with $\mathrm{Et}_{2} \mathrm{O}$. The combined organic extracts were washed with brine, dried over $\mathrm{MgSO}_{4}$, filtered, and concentrated under reduced pressure. Purification by column chromatography ( $5 \%$ EtOAc/hexanes) afforded 
$3.272 \mathrm{~g}(97 \%)$ of alcohol $\mathbf{8}$ and its syn diastereomer as a 96:4 inseparable mixture. $\mathrm{R}_{\mathrm{f}}=$ 0.79 (25\% EtOAc/hexanes); $[\alpha]^{20}{ }_{\mathrm{D}}=+14.1$ (c 0.9, $\mathrm{CHCl}_{3}$ ); IR (film) 3580, 3326, 2977, $2857,2173 \mathrm{~cm}^{-1} ;{ }^{1} \mathrm{H}$ NMR $\left(300 \mathrm{MHz}, \mathrm{CDCl}_{3}\right) 3.68(1 \mathrm{H}, \mathrm{dd}, J=3.6,6.0 \mathrm{~Hz}), 3.38(1 \mathrm{H}, \mathrm{t}$, $J=5.4 \mathrm{~Hz}), 2.73(2 \mathrm{H}, \mathrm{m}), 2.06(1 \mathrm{H}, \mathrm{d}, J=2.4 \mathrm{~Hz}), 2.03(1 \mathrm{H}, \mathrm{m}), 1.79(1 \mathrm{H}, \mathrm{br} \mathrm{s}), 1.22$ $(3 \mathrm{H}, \mathrm{d}, J=6.9 \mathrm{~Hz}), 1.20(3 \mathrm{H}, \mathrm{d}, J=7.2 \mathrm{~Hz}), 1.05(3 \mathrm{H}, \mathrm{d}, J=6.9 \mathrm{~Hz}), 0.91(9 \mathrm{H}, \mathrm{s}), 0.15$ $(9 \mathrm{H}, \mathrm{s}), 0.12(3 \mathrm{H}, \mathrm{s}), 0.07(3 \mathrm{H}, \mathrm{s}) ;{ }^{13} \mathrm{C} \mathrm{NMR}\left(75.5 \mathrm{MHz}, \mathrm{CDCl}_{3}\right)$ 107.39, 87.35, 76.40, 74.92, 70.12, 40.72, 32.15, 30.88, 26.07, 18.36, 17.78, 17.44, 9.67, 0.12, -3.74, -4.03. Anal. Calcd for $\mathrm{C}_{21} \mathrm{H}_{40} \mathrm{O}_{2} \mathrm{Si}_{2}$ : C, 66.25; H, 10.59. Found: C, 66.29; H, 10.41 .

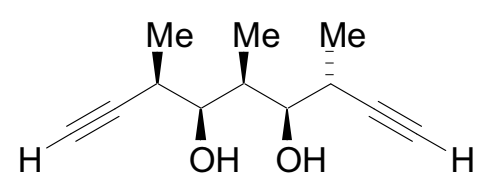

$(3 R, 4 R, 5 R, 6 S, 7 R)$-(-)-3,5,7-Trimethylnonan-1,8-diyne(1.0M in THF, $11.0 \mathrm{~mL}, 11.0 \mathrm{mmol})$. After $3 \mathrm{~h}$ the reaction was judged to be complete by TLC and was quenched by the addition of saturated aqueous $\mathrm{NH}_{4} \mathrm{Cl}(20 \mathrm{~mL})$ and EtOAc $(10 \mathrm{~mL})$. The layers were separated, and the aqueous layer was extracted with EtOAc. The combined organic extracts were washed with brine, dried over $\mathrm{MgSO}_{4}$, filtered, and concentrated under reduced pressure. Purification by column chromatography ( $25 \%$ EtOAc/hexanes) afforded $0.415 \mathrm{~g}(98 \%)$ of a yellow oil. $\mathrm{R}_{\mathrm{f}}=0.25$ (25\% EtOAc/hexanes); $[\alpha]^{20}{ }_{D}=-2.3\left(c 1.1, \mathrm{CHCl}_{3}\right)$; IR (film) 3315, 3266, 2976, 2951, $2871 \mathrm{~cm}^{-1} ;{ }^{1} \mathrm{H}$ NMR $\left(300 \mathrm{MHz}, \mathrm{CDCl}_{3}\right) \_3.65(2 \mathrm{H}, \mathrm{dq}, J=0.9,9.0 \mathrm{~Hz}), 2.68(1 \mathrm{H}, \mathrm{m})$, $2.56(1 \mathrm{H}, \mathrm{m}), 2.30(1 \mathrm{H}, \mathrm{m}), 2.20(1 \mathrm{H}, \mathrm{d}, J=2.4 \mathrm{~Hz}), 2.08(1 \mathrm{H}, \mathrm{d}, J=2.4 \mathrm{~Hz}), 1.32(3 \mathrm{H}$, d, $J=6.9 \mathrm{~Hz}), 1.18(3 \mathrm{H}, \mathrm{d}, J=6.9 \mathrm{~Hz}), 0.93(3 \mathrm{H}, \mathrm{d}, J=7.2 \mathrm{~Hz}) ;{ }^{13} \mathrm{C} \mathrm{NMR} \_(75.5 \mathrm{MHz} \text {, }$ $\left.\mathrm{CDCl}_{3}\right)$ 86.04, 85.65, 79.79, 79.40, 71.30, 70.19, 35.76, 31.26, 30.18, 17.80, 16.76, 4.26. Anal. Calcd for $\mathrm{C}_{12} \mathrm{H}_{18} \mathrm{O}_{2}$ : C, 74.19; H, 9.34. Found: C, 74.02; H, 9.42.

\section{$(3 R, 4 R, 5 R, 6 S, 7 R)-(-)-3,5,7-$ Trimethyl-4,6-}

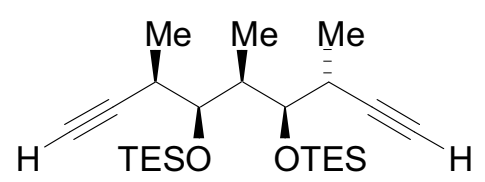
bis(triethylsilyloxy)nonan-1,8-diyne (10). To a stirring solution of diol 9 (0.254 g, $1.30 \mathrm{mmol})$ in $\mathrm{CH}_{2} \mathrm{Cl}_{2}(13 \mathrm{~mL})$ was added 2,6-lutidine $(0.61 \mathrm{~mL}, 5.2 \mathrm{mmol})$. The reaction 
was cooled to $0{ }^{\circ} \mathrm{C}$, and TESOTf $(0.65 \mathrm{~mL}, 2.9 \mathrm{mmol})$ was subsequently added. After 2 $\mathrm{h}$, the reaction was quenched by addition of $\mathrm{NH}_{4} \mathrm{Cl}(20 \mathrm{~mL})$ and $\mathrm{Et}_{2} \mathrm{O}(10 \mathrm{~mL})$. The layers were separated and the aqueous layer was washed with $\mathrm{Et}_{2} \mathrm{O}$. The combined organic extracts were washed with brine, dried over $\mathrm{MgSO}_{4}$, filtered, and concentrated under reduced pressure. Purification by column chromatography ( $0.5 \%$ EtOAc/hexanes) afforded $0.557 \mathrm{~g}(100 \%)$ of $\mathbf{1 0}$ as a yellow oil. $\mathrm{R}_{\mathrm{f}}=0.90(25 \% \mathrm{EtOAc} /$ hexanes $) ;[\alpha]^{20}{ }_{\mathrm{D}}=$ -0.2 (c 2.78, $\mathrm{CHCl}_{3}$ ); IR (film) 3327, 2960, $2890 \mathrm{~cm}^{-1} ;{ }^{1} \mathrm{H} \mathrm{NMR}\left(300 \mathrm{MHz}, \mathrm{CDCl}_{3}\right)_{-}$ $3.77(1 \mathrm{H}, \mathrm{dt}, J=1.2,5.4 \mathrm{~Hz}), 3.57(1 \mathrm{H}, \mathrm{dt}, J=1.2,4.5 \mathrm{~Hz}), 2.70(2 \mathrm{H}, \mathrm{m}), 2.06(3 \mathrm{H}, \mathrm{m})$, $1.19(6 \mathrm{H}, \mathrm{qd}, J=1.5,7.2 \mathrm{~Hz}), 0.94-1.03(21 \mathrm{H}, \mathrm{m}), 0.67(12 \mathrm{H}, \mathrm{q}, J=7.5 \mathrm{~Hz}) ;{ }^{13} \mathrm{C} \mathrm{NMR}$ _ (75.5 MHz, $\left.\mathrm{CDCl}_{3}\right)$ 87.79, 86.55, 76.16, 75.88, 70.22, 69.69, 40.30, 31.30, 30.17, 17.72, 15.71, 10.91, 7.09, 5.58. Anal. Calcd for $\mathrm{C}_{24} \mathrm{H}_{46} \mathrm{O}_{2} \mathrm{Si}_{2}: \mathrm{C}, 68.18 ; \mathrm{H}, 10.97$. Found: C, 68.36; H, 11.10 .

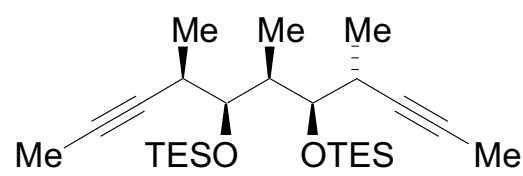

$(3 R, 4 R, 5 R, 6 S, 7 R)-(+)-4,6,8-T r i m e t h y l-5,7-$ bis(triethylsilyloxy)undecan-2,9-diyne (11). To a stirring solution of diyne $10(0.557 \mathrm{~g}, 1.30 \mathrm{mmol})$ in THF $(13 \mathrm{~mL})$ at $-78^{\circ} \mathrm{C}$ was added $\mathrm{BuLi}(2.4 \mathrm{M}$ in hexanes, $1.7 \mathrm{~mL}, 3.9 \mathrm{mmol})$. After $1 \mathrm{~h},(\mathrm{MeO})_{2} \mathrm{SO}_{2}(0.37 \mathrm{~mL}, 3.9 \mathrm{mmol})$ was added. After $15 \mathrm{~min}$, the reaction mixture was warmed to $0{ }^{\circ} \mathrm{C}$ for $1 \mathrm{~h}$, and then warmed to room temperature overnight. The reaction was quenched with $\mathrm{NH}_{4} \mathrm{Cl}(20 \mathrm{~mL})$ and $\mathrm{Et}_{2} \mathrm{O}(10$ $\mathrm{mL}$ ) and the layers were separated. The aqueous layers were extracted with $\mathrm{Et}_{2} \mathrm{O}$ and the combined organic extracts were washed with brine, dried over $\mathrm{MgSO}_{4}$, filtered, and concentrated under reduced pressure. Purification by column chromatography (1\% EtOAc/hexanes) afforded $0.596 \mathrm{~g}(100 \%)$ of $\mathbf{1 1}$ as a yellow oil. $\mathrm{R}_{\mathrm{f}}=0.98(25 \%$ EtOAc/hexanes); $[\alpha]^{20}{ }_{D}=+4.9\left(c\right.$ 0.81, $\mathrm{CHCl}_{3}$ ); IR (film) 2968, 2925, $2881 \mathrm{~cm}^{-1} ;{ }^{1} \mathrm{H}$ NMR $\left(300 \mathrm{MHz}, \mathrm{CDCl}_{3}\right)_{-} 3.72(1 \mathrm{H}, \mathrm{dd}, J=4.2,6.9 \mathrm{~Hz}), 3.54(1 \mathrm{H}, \mathrm{t}, J=4.8 \mathrm{~Hz}), 2.67$ $(1 \mathrm{H}, \mathrm{m}), 2.59(1 \mathrm{H}, \mathrm{m}), 1.93(1 \mathrm{H}, \mathrm{m}), 1.79(6 \mathrm{H}, \mathrm{s}), 1.13-1.07(6 \mathrm{H}, \mathrm{m}), 1.00-0.95(18 \mathrm{H}$, m), 0.94-0.89 (3H, m), $0.67(12 \mathrm{H}, \mathrm{dq}, J=2.4,7.8 \mathrm{~Hz}) ;{ }^{13} \mathrm{C} \mathrm{NMR}_{-}\left(75.5 \mathrm{MHz}, \mathrm{CDCl}_{3}\right)$ 83.29, 82.01, 77.17, 76.32, 76.08, 39.64, 31.80, 29.82, 17.72, 15.20, 10.89, 7.11, 5.56, $5.52,3.72,3.61$. 


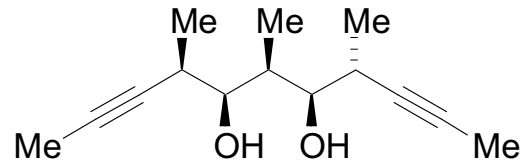

$(4 R, 5 R, 6 R, 7 S, 8 R)-(-)-4,6,8$-Trimethylundecan-2,9diyne-5,7-diol (12). To a solution of the bis-TES ether $11(2.68 \mathrm{~g}, 5.94 \mathrm{mmol})$ in $\mathrm{THF}(60 \mathrm{~mL})$ at $0{ }^{\circ} \mathrm{C}$ was added TBAF $(18 \mathrm{~mL}, 18 \mathrm{mmol})$. The reaction mixture was stirred for 30 minutes at $0{ }^{\circ} \mathrm{C}$, and then warmed to room temperature for $3 \mathrm{~h}$. The reaction was quenched with saturated aqueous $\mathrm{NH}_{4} \mathrm{Cl}(60 \mathrm{~mL})$ and extracted with ethyl acetate. The extract was washed with brine, dried over $\mathrm{MgSO}_{4}$, filtered, and concentrated under reduced pressure. The crude product was purified by chromatography on silica gel (10\% EtOAc/hexanes) to afford $1.29 \mathrm{~g}(98 \%)$ of a yellow solid. $\mathrm{R}_{\mathrm{f}}=0.35(25 \%$ EtOAc/hexanes $) ;[\alpha]^{20}{ }_{\mathrm{D}}=-11.89$ (c 2.00, $\mathrm{CHCl}_{3}$ ); IR (film) 3441, 2969, $1466 \mathrm{~cm}^{-1} ;{ }^{1} \mathrm{H} \mathrm{NMR}\left(300 \mathrm{MHz}, \mathrm{CDCl}_{3}\right) \_3.56$ $(1 \mathrm{H}, \mathrm{dd}, J=1.5,9.3 \mathrm{~Hz}), 3.54(1 \mathrm{H}, \mathrm{dd}, J=2.4,8.7 \mathrm{~Hz}), 3.01(2 \mathrm{H}, \mathrm{br} \mathrm{s}), 2.61(1 \mathrm{H}, \mathrm{dqq}, J$ $=2.4,6.6,6.9 \mathrm{~Hz}), 2.48(1 \mathrm{H}, \mathrm{dqq}, J=2.4,6.6,6.9 \mathrm{~Hz}), 2.24(1 \mathrm{H}, \mathrm{ddq}, J=2.1,6.9,7.2)$, $1.82(3 \mathrm{H}, \mathrm{d}, J=2.7 \mathrm{~Hz}), 1.77(3 \mathrm{H}, \mathrm{d}, J=2.4 \mathrm{~Hz}), 1.24(3 \mathrm{H}, \mathrm{d}, J=6.9), 1.12(3 \mathrm{H}, \mathrm{d}, J=$ $6.6 \mathrm{~Hz}), 0.89(3 \mathrm{H}, \mathrm{d}, J=6.9 \mathrm{~Hz}) ;{ }^{13} \mathrm{C} \mathrm{NMR}_{-}\left(75.5 \mathrm{MHz}, \mathrm{CDCl}_{3}\right) 80.98,80.10,80.05$, 79.95, 79.03, 77.44, 35.80, 31.40, 30.36, 18.16, 17.23, 4.36, 3.49. Anal. Calcd for $\mathrm{C}_{14} \mathrm{H}_{22} \mathrm{O}_{2}$ : C, 75.63; H, 9.97. Found: C, 75.35; H, 10.05.

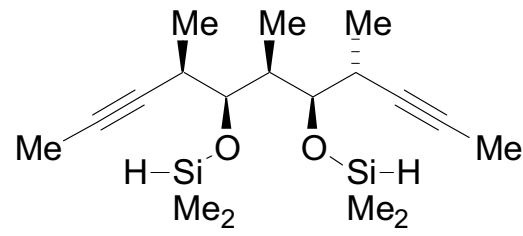

reaction mixture was stirred at room temperature until the solid diol dissolved, and then was heated to $80^{\circ} \mathrm{C}$. After $6 \mathrm{hr}$, the reaction mixture was cooled to room temperature and concentrated via rotary evaporation to remove excess silazane. The crude oil was heated to $60^{\circ} \mathrm{C}$ under high vacuum $(0.03 \mathrm{mmHg})$ overnight. The resulting viscous oil was used in the next step without purification. IR 2977, $2121 \mathrm{~cm}^{-1} ;{ }^{1} \mathrm{H}$ NMR $(300 \mathrm{MHz}$, $\left.\mathrm{CDCl}_{3}\right)_{-} 4.73(1 \mathrm{H}, \mathrm{m}), 4.69(1 \mathrm{H}, \mathrm{m}), 3.62(1 \mathrm{H}, \mathrm{dd}, J=4.5,6.9 \mathrm{~Hz}), 3.43(1 \mathrm{H}, \mathrm{dd}, J=$ $4.5,6.9 \mathrm{~Hz}), 2.71(1 \mathrm{H}, \mathrm{m}), 2.58(1 \mathrm{H}, \mathrm{m}), 2.24(1 \mathrm{H}, \mathrm{dq}, J=4.2,7.2 \mathrm{~Hz}), 1.80(3 \mathrm{H}, \mathrm{d}, J=$
$(4 R, 5 R, 6 R, 7 S, 8 R)-5,7-B i s($ dimethylsilyloxy)-4,6,8trimethylundecan-2,9-diyne (13). A dry flask was charged with diol 12 (0.219 g, $0.99 \mathrm{mmol})$, a stir bar, and tetramethyldisilazane $(3.5 \mathrm{~mL}, 19.7 \mathrm{mmol})$. The 
$2.4 \mathrm{~Hz}), 1.77(3 \mathrm{H}, \mathrm{d}, J=2.4 \mathrm{~Hz}), 1.15(3 \mathrm{H}, \mathrm{d}, J=6.9 \mathrm{~Hz}), 1.13(3 \mathrm{H}, \mathrm{d}, J=6.9 \mathrm{~Hz}), 0.92$ $(3 \mathrm{H}, \mathrm{d}, J=6.9 \mathrm{~Hz}), 0.26(3 \mathrm{H}, \mathrm{dd}, J=2.7,3.0 \mathrm{~Hz}), 0.22(3 \mathrm{H}, \mathrm{dd}, J=2.7,5.4 \mathrm{~Hz})$.

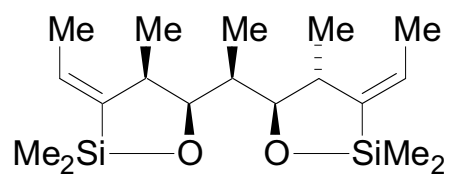

$(4 R, 5 R, 6 R, 7 S, 8 R)$-Bis-Siloxane (14). The residue from the previous step was dissolved in THF (4 mL), and $\mathrm{H}_{2} \mathrm{PtCl}_{6}$ ( $0.05 \mathrm{M}$ in THF, $0.1 \mathrm{~mL}, 4.95$ mol) was added. The yellow solution was heated to $75^{\circ} \mathrm{C}$. After $5 \mathrm{~h}$ the hydrosilylation was judged complete by IR analysis ( $\mathrm{Si}-\mathrm{H}, 2121 \mathrm{~cm}^{-1}$ ). The mixture was diluted with ether and filtered through Celite 521. The mother liquor was concentrated under reduced pressure to afford $0.323 \mathrm{~g}$ (97\%) of 14 as a yellow oil that was used without further purification. IR 2977, $1256 \mathrm{~cm}^{-}$ '; ${ }^{1} \mathrm{H}$ NMR (300 MHz, $\left.\mathrm{CDCl}_{3}\right) \delta 5.81(2 \mathrm{H}, \mathrm{m}), 4.03(1 \mathrm{H}, \mathrm{t}, J=2.1 \mathrm{~Hz}), 3.67(2 \mathrm{H}, \mathrm{dd}, J=$ 3.9, $9.6 \mathrm{~Hz}), 2.99(1 \mathrm{H}, \mathrm{m}), 2.67(1 \mathrm{H}, \mathrm{q}, J=7.2 \mathrm{~Hz}), 1.76(3 \mathrm{H}, \mathrm{d}, J=6.6 \mathrm{~Hz}), 1.75(3 \mathrm{H}, \mathrm{d}$, $J=6.3 \mathrm{~Hz}), 1.12(3 \mathrm{H}, \mathrm{d}, J=7.5 \mathrm{~Hz}), 0.91(3 \mathrm{H}, \mathrm{d}, J=6.6 \mathrm{~Hz}), 0.89(3 \mathrm{H}, \mathrm{d}, J=7.2 \mathrm{~Hz})$, $0.24(3 \mathrm{H}, \mathrm{d}, J=3.0 \mathrm{~Hz}), 0.22(3 \mathrm{H}, \mathrm{s}), 0.15(3 \mathrm{H}, \mathrm{s}), 0.07(3 \mathrm{H}, \mathrm{s})$.

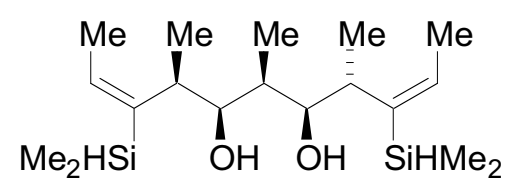

$(4 R, 5 R, 6 R, 7 S, 8 R)$-Diol (15). A solution of bissiloxane $14(53.6 \mathrm{mg}, 0.16 \mathrm{mmol})$ in toluene $(1.5 \mathrm{~mL})$ was cooled to $-78^{\circ} \mathrm{C}$ for 15 minutes. DIBAL-H (3 mL, $1.0 \mathrm{M}$ in toluene, $3 \mathrm{mmol}$ ) was added and the reaction stirred for $1 \mathrm{~h}$. The reaction mixture was poured into Rochelle's salts at $0{ }^{\circ} \mathrm{C}$, stirred overnight, and extracted with EtOAc. The extract was washed with brine, dried over $\mathrm{MgSO}_{4}$, filtered, and concentrated under reduced pressure. The crude product was purified by chromatography on silica gel (10\% EtOAc/hexanes) to afford $51.8 \mathrm{mg}(96 \%)$ of a clear oil. ${ }^{1} \mathrm{H}$ NMR (300 $\left.\mathrm{MHz}, \mathrm{CDCl}_{3}\right)_{-} 5.83(2 \mathrm{H}, \mathrm{m}), 4.62(2 \mathrm{H}, \mathrm{s}), 4.03(1 \mathrm{H}, \mathrm{m}), 3.84(1 \mathrm{H}, \mathrm{m}), 2.99(1 \mathrm{H}, \mathrm{m})$, $2.91(1 \mathrm{H}, \mathrm{m}), 2.65(1 \mathrm{H}, \mathrm{m}), 1.76(3 \mathrm{H}, \mathrm{d}, J=6.6 \mathrm{~Hz}), 1.75(3 \mathrm{H}, \mathrm{d}, J=6.3 \mathrm{~Hz}), 1.12(3 \mathrm{H}$, d, $J=7.5 \mathrm{~Hz}), 0.91(3 \mathrm{H}, \mathrm{d}, J=6.6 \mathrm{~Hz}), 0.89(3 \mathrm{H}, \mathrm{d}, J=7.2 \mathrm{~Hz}), 0.24(3 \mathrm{H}, \mathrm{d}, J=3.0 \mathrm{~Hz})$, $0.22(3 \mathrm{H}, \mathrm{s}), 0.15(3 \mathrm{H}, \mathrm{s}), 0.07(3 \mathrm{H}, \mathrm{s})$. 


\section{Hydride Siloxane Cleavage Model System}

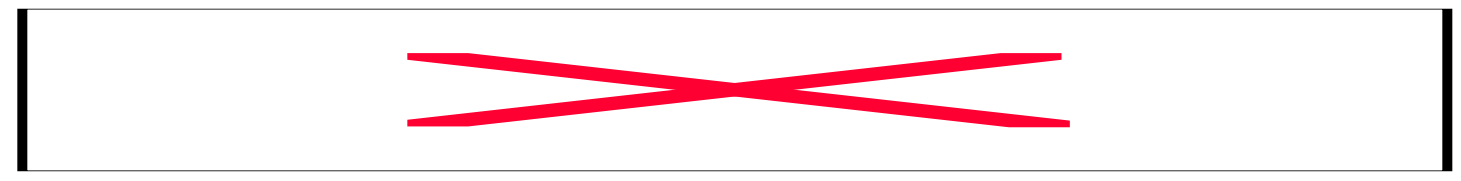

Alcohol (II). To a solution of alcohol $\mathbf{I}^{1}(300 \mathrm{mg}, 1.40 \mathrm{mmol})$ in
toluene $(7 \mathrm{~mL})$ at $0^{\circ} \mathrm{C}$ was added DIBAL-H $(14 \mathrm{~mL}, 1.0 \mathrm{M}$ in toluene, cooled to room temperature and poured into Rochelle's salts. Stirring overnight was followed by extraction with EtOAc. The extract was washed with brine, dried over $\mathrm{MgSO}_{4}$, filtered, and concentrated under reduced pressure. The crude product was purified by chromatography on silica gel (10\% EtOAc/hexanes) to afford $196 \mathrm{mg}(65 \%)$ of clear oil. ${ }^{1} \mathrm{H}$ NMR $\left(300 \mathrm{MHz}, \mathrm{CDCl}_{3}\right)_{-} 7.35(5 \mathrm{H}, \mathrm{m}), 6.19(1 \mathrm{H}, \mathrm{q}), 4.78(1 \mathrm{H}, \mathrm{q}), 4.11$ $(1 \mathrm{H}, \mathrm{m}), 2.65$ (2H, ddd), $1.96(1 \mathrm{H}$, br s), $1.61(3 \mathrm{H}, \mathrm{d}), 0.14(6 \mathrm{H}, \mathrm{m})$.

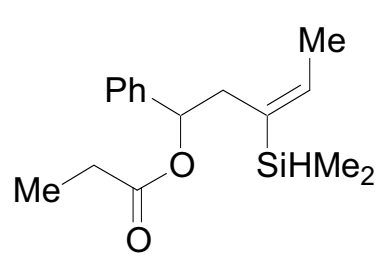

Ester (III). To a solution of alcohol II $(45.0 \mathrm{mg}, 0.20 \mathrm{mmol})$ in $\mathrm{CH}_{2} \mathrm{Cl}_{2}$ was added DMAP $(5 \mathrm{mg}, 40 \mu \mathrm{mol})$. The reaction mixture was stirred until the DMAP dissolved and was then cooled to $0{ }^{\circ} \mathrm{C}$. Propionic anhydride $(0.05 \mathrm{~mL}, 0.40 \mathrm{mmol})$ and $\mathrm{NEt}_{3}(0.08 \mathrm{~mL}, 0.60 \mathrm{mmol})$ were added and the reaction mixture stirred at $0{ }^{\circ} \mathrm{C}$ for $20 \mathrm{~h}$. The reaction was quenched with saturated aqueous $\mathrm{NH}_{4} \mathrm{Cl}$ and extracted with $\mathrm{CH}_{2} \mathrm{Cl}_{2}$. The extract was washed with brine, dried over $\mathrm{MgSO}_{4}$, filtered, and concentrated under reduced pressure. The crude product was purified by chromatography on silica gel $(10 \%$ EtOAc/hexanes) to afford $50 \mathrm{mg}(89 \%)$ of a clear oil. ${ }^{1} \mathrm{H}$ NMR $\left(300 \mathrm{MHz}, \mathrm{CDCl}_{3}\right)_{-}$ $7.19(5 \mathrm{H}, \mathrm{m}), 6.03(1 \mathrm{H}, \mathrm{q}), 5.79(1 \mathrm{H}, \mathrm{q}), 4.01(1 \mathrm{H}, \mathrm{m}), 2.73(2 \mathrm{H}, \mathrm{ddd}), 2.38(2 \mathrm{H}, \mathrm{dd})$, $1.61(3 \mathrm{H}, \mathrm{d}), 1.14(3 \mathrm{H}, \mathrm{t}), 0.14(6 \mathrm{H}, \mathrm{m})$.<smiles>CCC(=O)CC(=O)c1ccccc1</smiles>

Diketone (IV). To silane III (50.0 mg, $0.20 \mathrm{mmol})$ in MeOH/THF (2:1, $3 \mathrm{~mL}$ ) was added $\mathrm{KF}$ (107 $\mathrm{mg}, 2 \mathrm{mmol})$. The reaction mixture was 
stirred for $15 \mathrm{~min}$, then cooled to $0{ }^{\circ} \mathrm{C} . \mathrm{H}_{2} \mathrm{O}_{2}(1.7 \mathrm{~mL}, 15 \mathrm{mmol})$ and $\mathrm{KHCO}_{3}(116 \mathrm{mg}$, $1.0 \mathrm{mmol}$ ) were added, and the reaction was heated to $65^{\circ} \mathrm{C}$. After $2 \mathrm{~h}$, the reaction was cooled to $0{ }^{\circ} \mathrm{C}$, carefully quenched with methanolic $\mathrm{Na}_{2} \mathrm{~S}_{2} \mathrm{O}_{4}(10 \%)$, and diluted with $\mathrm{Et}_{2} \mathrm{O}$. Extraction with $\mathrm{Et}_{2} \mathrm{O}$ was followed by washing with brine, drying over $\mathrm{MgSO}_{4}$, and concentration under reduced pressure. The crude product was purified by chromatography on silica gel (10\% EtOAc/hexanes) to afford $25 \mathrm{mg}(78 \%)$ of a yellow oil. ${ }^{1} \mathrm{H}$ NMR (300 MHz, $\left.\mathrm{CDCl}_{3}\right)$ _ $7.35(5 \mathrm{H}, \mathrm{m}), 3.99(1 \mathrm{H}, \mathrm{s}), 3.55(1 \mathrm{H}, \mathrm{s}), 2.49(2 \mathrm{H}, \mathrm{m})$, $1.15(3 \mathrm{H}, \mathrm{t})$.

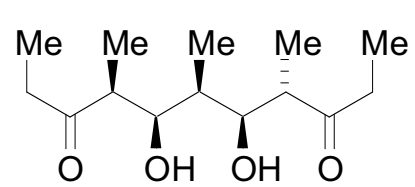

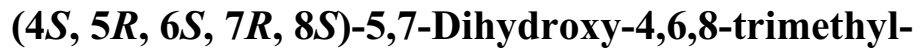

undecane-3,9-dione (16). To bis-siloxane 14 (0.252 g, 0.74

$\mathrm{mmol})$ in $\mathrm{MeOH} / \mathrm{THF}(2: 1,11.1 \mathrm{~mL})$ were added $\mathrm{KF}(0.43 \mathrm{~g}$, $7.4 \mathrm{mmol})$ and $\mathrm{KHCO}_{3}(0.74 \mathrm{~g}, 7.4 \mathrm{mmol})$ and the reaction mixture was cooled to $0{ }^{\circ} \mathrm{C}$ for 15 minutes. $\mathrm{H}_{2} \mathrm{O}_{2}(11 \mathrm{~mL}, 111 \mathrm{mmol})$ was added slowly by syringe. After three hours, the reaction was carefully quenched with methanolic $\mathrm{Na}_{2} \mathrm{~S}_{2} \mathrm{O}_{4}(10 \%)$ and diluted with EtOAc. Extraction with EtOAc followed by washing with brine, drying over $\mathrm{MgSO}_{4}$, and concentration under reduced pressure afforded $0.206 \mathrm{~g} \mathrm{(107 \% )}$ of crude $\beta$ hydroxy diketone which was carried on without purification. ${ }^{1} \mathrm{H}$ NMR $(300 \mathrm{MHz}$, $\left.\mathrm{CDCl}_{3}\right)_{-} 4.49(1 \mathrm{H}, \mathrm{t}, J=2.4 \mathrm{~Hz}), 3.45(2 \mathrm{H}, \mathrm{t}, J=3.0 \mathrm{~Hz}), 2.72(1 \mathrm{H}, \mathrm{m}), 2.66(1 \mathrm{H}, \mathrm{dq}, J$ $=7.2,17.7 \mathrm{~Hz}), 2.42(1 \mathrm{H}, \mathrm{dq}, J=7.2,17.7 \mathrm{~Hz}), 2.16(1 \mathrm{H}, \mathrm{dq}, J=7.5 .14 .1 \mathrm{~Hz}), 2.11(1 \mathrm{H}$, qd, $J=3.3,7.5 \mathrm{~Hz}), 1.76(1 \mathrm{H}, \mathrm{m}), 1.70(1 \mathrm{H}, \mathrm{dq}, J=7.5,14.1 \mathrm{~Hz}), 1.32(3 \mathrm{H}, \mathrm{d}, J=7.5$ $\mathrm{Hz}), 1.07(3 \mathrm{H}, \mathrm{t}, J=7.2 \mathrm{~Hz}), 1.05(3 \mathrm{H}, \mathrm{d}, J=7.2 \mathrm{~Hz}), 0.98(3 \mathrm{H}, \mathrm{d}, J=6.9 \mathrm{~Hz}), 0.91(3 \mathrm{H}$, $\mathrm{t}, J=7.8 \mathrm{~Hz})$.

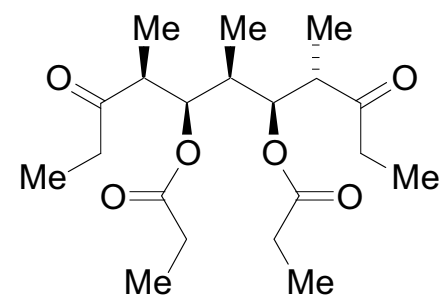

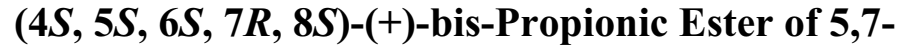
Dihydroxy-4,6,8-trimethyl-undecane-3,9-dione (17). To a solution of $\beta$-hydroxy diketone 16 (0.206 g, $0.80 \mathrm{mmol})$ and propionic acid $(0.30 \mathrm{~mL}, 4.0 \mathrm{mmol})$ in $\mathrm{CH}_{2} \mathrm{Cl}_{2}(8 \mathrm{~mL})$ at $0{ }^{\circ} \mathrm{C}$ was added DMAP $(0.109 \mathrm{~g}, 0.80 \mathrm{mmol})$ and DCC $(0.83 \mathrm{~g}, 4.0$ 
mmol). After $12 \mathrm{~h}$, the reaction mixture was filtered through a plug of Celite 521 and concentrated under reduced pressure. Chromatography on silica gel $(5 \%$

EtOAc/hexanes) afforded $0.126 \mathrm{~g}$ (46\% for 2 steps) of diketo diester $17 . \mathrm{R}_{\mathrm{f}}=0.58(25 \%$ EtOAc/hexanes); $[\alpha]^{20}{ }_{\mathrm{D}}=+70.2\left(c 0.79, \mathrm{CHCl}_{3}\right)$; IR (film) 2986, 2950, $1736 \mathrm{~cm}^{-1} ;{ }^{1} \mathrm{H}$ $\operatorname{NMR}\left(500 \mathrm{MHz}, \mathrm{CDCl}_{3}\right)_{-} 5.17(1 \mathrm{H}, \mathrm{dd}, J=2.5,9.5 \mathrm{~Hz}), 5.14(1 \mathrm{H}, \mathrm{dd}, J=3.5,9.0 \mathrm{~Hz})$, $3.30(1 \mathrm{H}, \mathrm{dq}, J=3.0,7.0 \mathrm{~Hz}), 2.99(1 \mathrm{H}, \mathrm{m}), 2.25-2.64(8 \mathrm{H}, \mathrm{m}), 2.07(1 \mathrm{H}, \mathrm{m}), 1.02-1.15$ $(15 \mathrm{H}, \mathrm{m}), 0.99(3 \mathrm{H}, \mathrm{t}, J=7.5 \mathrm{~Hz}), 0.91(3 \mathrm{H}, \mathrm{d}, J=6.5 \mathrm{~Hz}) ;{ }^{13} \mathrm{C} \mathrm{NMR} \_(125 \mathrm{MHz} \text {, }$ $\left.\mathrm{CDCl}_{3}\right)_{2} 212.01,73.65,73.10,47.56,46.31,35.74,35.45,34.26,27.59,27.46,13.84$, $9.55,9.26,9.11,7.70,7.54$.

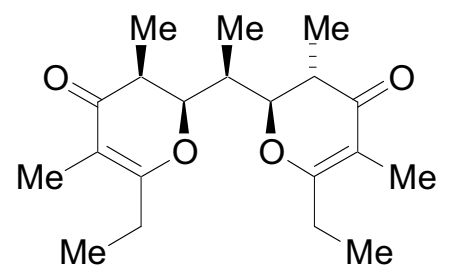

(-)-Membrenone C (18). To diester diketone 17 (25 mg, $0.06 \mathrm{mmol})$ in $\mathrm{CH}_{2} \mathrm{Cl}_{2}(5.4 \mathrm{~mL})$ at $-78^{\circ} \mathrm{C}$ was added Hunig's base $(0.15 \mathrm{~mL}, 0.86 \mathrm{mmol})$. After $15 \mathrm{~min}$ at $-78^{\circ} \mathrm{C}, \mathrm{TiCl}_{4}$ (1.0 $\mathrm{M}$ in $\mathrm{CH}_{2} \mathrm{Cl}_{2}, 0.55 \mathrm{~mL}, 0.55 \mathrm{mmol}$ ) was added over $1 \mathrm{~h}$.

After being stirred for an additional $45 \mathrm{~min}$ at $-78^{\circ} \mathrm{C}$, the reaction mixture was warmed to $-5{ }^{\circ} \mathrm{C}$ for $24 \mathrm{~h}$. The reaction was quenched with $\mathrm{NH}_{4} \mathrm{Cl}$ and extracted with $\mathrm{Et}_{2} \mathrm{O}$. The extract was washed with brine, dried over $\mathrm{MgSO}_{4}$, filtered, and concentrated under reduced pressure. The crude product was purified by chromatography on silica gel $(10 \%$ $\left.\mathrm{Et}_{2} \mathrm{O} / \mathrm{CH}_{2} \mathrm{Cl}_{2}\right)$ to afford $11.3 \mathrm{mg}(51 \%)$ of a yellow solid. $\mathrm{R}_{\mathrm{f}}=0.30\left(10 \% \mathrm{Et}_{2} \mathrm{O} / \mathrm{CH}_{2} \mathrm{Cl}_{2}\right)$; $[\alpha]^{20}{ }_{\mathrm{D}}=-22.6\left(\mathrm{c} \mathrm{0.85}, \mathrm{CHCl}_{3}\right)$ lit. $^{2}-28.2$ ( c $\left.0.46 \mathrm{CHCl}_{3}\right)$; IR (film) 2986, 2934, 2882, $1675,1615 \mathrm{~cm}^{-1} ;{ }^{1} \mathrm{H}$ NMR $\left(500 \mathrm{MHz}, \mathrm{CDCl}_{3}\right)_{-} 4.25(1 \mathrm{H}, \mathrm{dd}, J=2.5,10.0 \mathrm{~Hz}), 3.89$ $(1 \mathrm{H}, \mathrm{dd}, J=2.0,13.5 \mathrm{~Hz}), 2.51(1 \mathrm{H}, \mathrm{m}), 2.22-2.46(5 \mathrm{H}, \mathrm{m}), 2.21(1 \mathrm{H}, \mathrm{m}), 1.74(3 \mathrm{H}, \mathrm{s})$, $1.71(3 \mathrm{H}, \mathrm{s}), 1.19(3 \mathrm{H}, \mathrm{d}, J=7.0 \mathrm{~Hz}), 1.18(3 \mathrm{H}, \mathrm{t}, J=8.0 \mathrm{~Hz}), 1.10(3 \mathrm{H}, \mathrm{d}, J=7.0 \mathrm{~Hz})$, $1.07(3 \mathrm{H}, \mathrm{t}, J=7.5 \mathrm{~Hz}), 1.02(3 \mathrm{H}, \mathrm{d}, J=7.5 \mathrm{~Hz}) ;{ }^{13} \mathrm{C} \mathrm{NMR}_{-}\left(125 \mathrm{MHz}, \mathrm{CDCl}_{3}\right)$ 197.10, 194.56, 173.71, 172.48, 108.66, 107.71, 81.69, 80.94, 40.44, 39.92, 34.67, 25.42, $10.91,10.81,9.78,9.32,9.25,9.10$.

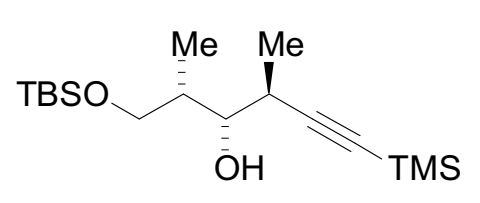

$(2 S, 3 S, 4 S)-(-)-1-($ tert-Butyldimethylsilyloxy)-2,4dimethyl-6-trimethylsilyl-5-hexyn-3-ol (19). To a stirring solution of (2S)-3-(tert-butyldimethylsilyloxy)-2- 
methylpropanal 2 (1.23 g, $6.0 \mathrm{mmol})$ in THF/HMPA $(3: 1,33 \mathrm{~mL})$ was added Pd(OAc) $(0.081 \mathrm{~g}, 0.36 \mathrm{mmol})$. The reaction mixture was cooled to $0{ }^{\circ} \mathrm{C}$ and $\mathrm{PPh}_{3}(0.095 \mathrm{~g}, 0.36$ mmol) was added. Upon complete dissolution of the $\mathrm{PPh}_{3}$, pulverized InI (2.02 g, 8.0 mmol) was added, followed by the mesylate of $(R)$-4-TMS-3-butyn-2-ol (1.85 g, 8.4 mmol). After stirring at $0{ }^{\circ} \mathrm{C}$ for $2 \mathrm{~h}$, the reaction mixture was quenched by pouring into a stirring solution of $\mathrm{NH}_{4} \mathrm{Cl}(25 \mathrm{~mL})$ and $\mathrm{Et}_{2} \mathrm{O}(25 \mathrm{~mL})$. The layers were separated and the aqueous layer was washed with $\mathrm{Et}_{2} \mathrm{O}$. The combined organic extracts were washed with brine, dried over $\mathrm{MgSO}_{4}$, filtered, and concentrated under reduced pressure. Purification by column chromatography (5\% EtOAc/hexanes) afforded $1.49 \mathrm{~g}(75 \%)$ of alcohol 19. $\mathrm{R}_{\mathrm{f}}=0.75(25 \%$ EtOAc/hexanes $) ;[\alpha]^{20}=-4.8\left(c 1.21, \mathrm{CHCl}_{3}\right) ; \mathrm{IR}$ (film) 3510, 2968, 2182, $1946 \mathrm{~cm}^{-1}$; ${ }^{1} \mathrm{H}$ NMR $\left(300 \mathrm{MHz}, \mathrm{CDCl}_{3}\right)_{-} 3.63(2 \mathrm{H}, \mathrm{m}), 3.51(1 \mathrm{H}, \mathrm{s})$, $2.69(1 \mathrm{H}, \mathrm{m}), 2.33(1 \mathrm{H}, \mathrm{d}, J=4.5 \mathrm{~Hz}), 1.78(1 \mathrm{H}, \mathrm{m}), 1.17(3 \mathrm{H}, \mathrm{d}, J=7.2 \mathrm{~Hz}), 0.94(3 \mathrm{H}$, $\mathrm{d}, J=6.9 \mathrm{~Hz}), 0.90(9 \mathrm{H}, \mathrm{s}), 0.15(9 \mathrm{H}, \mathrm{s}), 0.06(6 \mathrm{H}, \mathrm{s}) ;{ }^{13} \mathrm{C} \mathrm{NMR}_{-}\left(75.5 \mathrm{MHz}, \mathrm{CDCl}_{3}\right)$ 108.49, 87.00, 75.26, 66.66, 38.07, 31.88, 25.87, 18.24, 17.66, 10.43, 0.14, -0.91, -5.50. Anal. Calcd for $\mathrm{C}_{17} \mathrm{H}_{36} \mathrm{O}_{2} \mathrm{Si}_{2}$ : C, 62.13; H, 11.04. Found: C, 62.33; H, 10.92 .

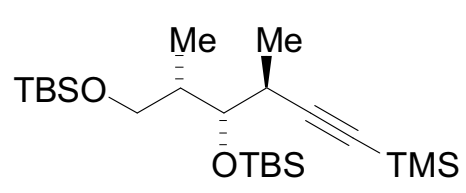

\section{$(2 S, 3 S, 4 S)-(+)-4,6-B i s(t e r t-b u t y l d i m e t h y l s i l y l o x y)-3,5-$} dimethyl-1-trimethylsilyl-1-hexyne (20). To a solution of alcohol 19 (0.77 g, $2.30 \mathrm{mmol})$ in $\mathrm{CH}_{2} \mathrm{Cl}_{2}(25 \mathrm{~mL})$ at $0{ }^{\circ} \mathrm{C}$ was added 2,6-lutidine $(0.40 \mathrm{~mL}, 5.5 \mathrm{mmol})$ and TBSOTf

$(0.70 \mathrm{~mL}, 3.0 \mathrm{mmol})$. The mixture was allowed to warm to room temperature and stirred for $1.5 \mathrm{~h}$. The reaction was quenched with $\mathrm{H}_{2} \mathrm{O}(25 \mathrm{~mL})$, and the aqueous layer was extracted with $\mathrm{CH}_{2} \mathrm{Cl}_{2}$, dried over $\mathrm{MgSO}_{4}$, filtered, and concentrated under reduced pressure. Purification by chromatography on silica gel (1\% EtOAc/hexanes) afforded $1.00 \mathrm{~g}(97 \%)$ of a colorless oil. $\mathrm{R}_{\mathrm{f}}=0.93(25 \%$ EtOAc/hexanes $) ;[\alpha]^{20}{ }_{\mathrm{D}}=+2.9(c 1.73$, $\mathrm{CHCl}_{3}$ ); IR (film) 2960, 2174, $1929 \mathrm{~cm}^{-1} ;{ }^{1} \mathrm{H} \mathrm{NMR}\left(300 \mathrm{MHz}, \mathrm{CDCl}_{3}\right)_{-} 3.76(1 \mathrm{H}, \mathrm{s})$, $3.49(2 \mathrm{H}, \mathrm{m}), 2.64(1 \mathrm{H}, \mathrm{m}), 1.91(1 \mathrm{H}, \mathrm{m}), 1.17(3 \mathrm{H}, \mathrm{d}, J=7.2 \mathrm{~Hz}), 0.92(9 \mathrm{H}, \mathrm{s}), 0.91$ $(9 \mathrm{H}, \mathrm{s}), 0.86(3 \mathrm{H}, \mathrm{d}, J=5.4 \mathrm{~Hz}), 0.14(9 \mathrm{H}, \mathrm{s}), 0.09(6 \mathrm{H}, \mathrm{d}, J=7.5 \mathrm{~Hz}), 0.05(6 \mathrm{H}, \mathrm{s}) ;{ }^{13} \mathrm{C}$ NMR_(75.5 MHz, $\left.\mathrm{CDCl}_{3}\right)$ 110.28, 85.72, 81.49, 74.12, 65.84, 39.58, 32.80, 25.97, 
18.42, 18.26, 17.88, 11.61, 0.16, -3.98, -4.18, -5.23, -5.27. Anal. Calcd for $\mathrm{C}_{23} \mathrm{H}_{50} \mathrm{O}_{2} \mathrm{Si}_{3}$ : C, 62.37; H, 11.38. Found: C, 62.58; H, 11.44.

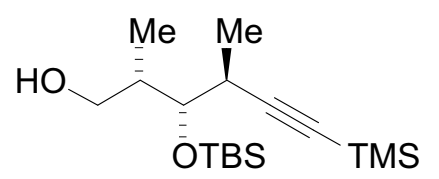

(2S, 3S, 4S)-(+)-3-(tert-Butyldimethylsilyloxy)-2,4-dimethyl6-trimethylsilyl-5-hexyn-1-ol (21). Bis-silyl ether 20 (0.852 g, $1.92 \mathrm{mmol})$ was taken up in $95 \% \mathrm{EtOH}(20 \mathrm{~mL})$ and PPTS (120 mg, $0.50 \mathrm{mmol}$ ) was added at room temperature. After the reaction was judged complete by TLC (approximately 48 to $60 \mathrm{~h}$ ), brine $(25 \mathrm{~mL}$ ) was added and the mixture was extracted with ether, dried over $\mathrm{MgSO}_{4}$, filtered, and concentrated under reduced pressure. Purification by chromatography on silica gel (10\% EtOAc/hexanes) afforded $0.568 \mathrm{~g}(90 \%)$ of a yellow oil. $\mathrm{R}_{\mathrm{f}}=0.45(25 \% \mathrm{EtOAc} /$ hexanes $) ;[\alpha]^{20}{ }_{\mathrm{D}}=+8.7(c$ 1.77, $\left.\mathrm{CHCl}_{3}\right)$; IR (film) 3362, 2960, 2165, $1945 \mathrm{~cm}^{-1} ;{ }^{1} \mathrm{H} \mathrm{NMR}\left(300 \mathrm{MHz}, \mathrm{CDCl}_{3}\right) \_3.64(2 \mathrm{H}$, m), $3.62(1 \mathrm{H}, \mathrm{m}), 2.62(1 \mathrm{H}, \mathrm{dd}, J=3.3,6.9 \mathrm{~Hz}), 2.49(1 \mathrm{H}, \mathrm{br} \mathrm{s}), 1.91(1 \mathrm{H}, \mathrm{m}), 1.17(3 \mathrm{H}$, d, $J=7.2 \mathrm{~Hz}), 0.89(9 \mathrm{H}, \mathrm{s}), 0.87(3 \mathrm{H}, \mathrm{d}, J=6.9 \mathrm{~Hz}), 0.11(6 \mathrm{H}, \mathrm{s}), 0.06(6 \mathrm{H}, \mathrm{d}, J=11.7$ $\mathrm{Hz}) ;{ }^{13} \mathrm{C} \mathrm{NMR}_{-}\left(75.5 \mathrm{MHz}, \mathrm{CDCl}_{3}\right) \quad 109.49,86.31,76.56,64.93,39.84,31.04,25.87$, 25.64, 18.14, 18.08, 13.04, -0.05, -0.41, -3.63, -4.22, -4.50. Anal. Calcd for $\mathrm{C}_{17} \mathrm{H}_{36} \mathrm{O}_{2} \mathrm{Si}_{2}$ : C, 62.13; H, 11.04. Found: C, 62.07; H, 11.09 .

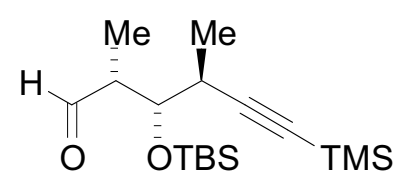

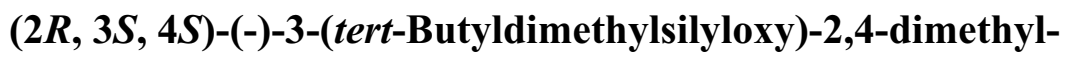
6-trimethylsilyl-5-hexynal (22). To a stirring solution of oxalyl chloride $(0.10 \mathrm{~mL}, 1.10 \mathrm{mmol})$ in $\mathrm{CH}_{2} \mathrm{Cl}_{2}(7.6 \mathrm{~mL})$ at $-78^{\circ} \mathrm{C}$ was added DMSO (0.16 mL, $2.3 \mathrm{mmol})$. After $15 \mathrm{~min}$, alcohol 21 (0.248 g, $0.76 \mathrm{mmol})$ in $\mathrm{CH}_{2} \mathrm{Cl}_{2}(1 \mathrm{~mL})$ was added. After $15 \mathrm{~min}$, triethylamine $(0.50 \mathrm{~mL}, 3.4 \mathrm{mmol})$ was added dropwise. The reaction mixture was stirred at $-78^{\circ} \mathrm{C}$ for $3 \mathrm{~h}$, and then warmed to $40{ }^{\circ} \mathrm{C}$. After $10 \mathrm{~min}$, the reaction was quenched with saturated $\mathrm{NaHCO}_{3}(20 \mathrm{~mL})$, and warmed to room temperature. The layers were separated and the aqueous layer was washed two times with $\mathrm{CH}_{2} \mathrm{Cl}_{2}$. The combined organic layers were washed with brine, dried over $\mathrm{MgSO}_{4}$, filtered, and concentrated under reduced pressure. The crude oil was triturated with $10 \%$ EtOAc/hexanes, filtered, and concentrated to afford $0.250 \mathrm{~g}(100 \%)$ 
of light yellow oil which was used without further purification. $\mathrm{R}_{\mathrm{f}}=0.88(25 \%$ EtOAc/hexanes); $[\alpha]^{20}{ }_{\mathrm{D}}=-29.3$ (c $0.91 \mathrm{CHCl}_{3}$ ); IR (film) 2968, 2855, 2165, $1736 \mathrm{~cm}^{-1}$; ${ }^{1} \mathrm{H}$ NMR (300 MHz, CDCl $)_{3}$ _ $9.89(1 \mathrm{H}, \mathrm{s}), 4.05(1 \mathrm{H}, \mathrm{t}, J=4.8 \mathrm{~Hz}), 2.69(1 \mathrm{H}, \mathrm{m}), 2.60$ $(1 \mathrm{H}, \mathrm{m}), 1.20(3 \mathrm{H}, \mathrm{d}, J=7.5 \mathrm{~Hz}), 1.12(3 \mathrm{H}, \mathrm{d}, J=7.2 \mathrm{~Hz}), 0.91(9 \mathrm{H}, \mathrm{s}), 0.13(6 \mathrm{H}, \mathrm{s}), 0.09$ $(6 \mathrm{H}, \mathrm{d}, J=13.8 \mathrm{~Hz}) ;{ }^{13} \mathrm{C} \mathrm{NMR}_{-}\left(75.5 \mathrm{MHz}, \mathrm{CDCl}_{3}\right)$ 203.56, 74.14, 50.67, 32.06, $25.77,18.15,17.16,9.69,-0.20,-4.36,-4.47$.

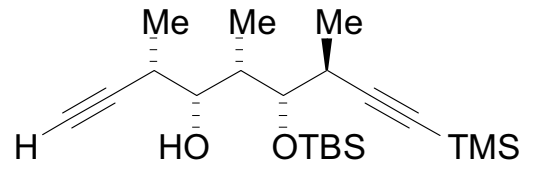

$(3 S, 4 S, 5 S, 6 R, 7 S)-(+)-4-($ tert-Butyldimethylsilyloxy)$\mathrm{g}, 0.62 \mathrm{mmol})$ and aldehyde $22(0.100 \mathrm{~g}, 0.31 \mathrm{mmol})$ in $\mathrm{CH}_{2} \mathrm{Cl}_{2}(3.1 \mathrm{~mL})$ at $-78^{\circ} \mathrm{C}$ was added $\mathrm{BF}_{3} \cdot \mathrm{OEt}_{2}(0.15 \mathrm{~mL}, 0.93 \mathrm{mmol})$ dropwise over $5 \mathrm{~min}$. After $1 \mathrm{~h}$ the reaction was quenched at $-78{ }^{\circ} \mathrm{C}$ by addition of $5 \mathrm{~mL}$ of $\mathrm{MeOH} / \mathrm{NaHCO}_{3}$ (sat.) $(5: 1, \mathrm{v} / \mathrm{v})$ and warmed to room temperature. The layers were separated and the aqueous layer was extracted with ether. The combined organic extracts were washed with brine, dried over $\mathrm{MgSO}_{4}$, filtered, and concentrated under reduced pressure. Purification by gradient elution chromatography on silica gel ( $0 \%-1 \%$ EtOAc/hexanes) afforded $0.089 \mathrm{~g}(76 \%)$ of a colorless oil. $\mathrm{R}_{\mathrm{f}}=0.73\left(25 \%\right.$ EtOAc/hexanes); $[\alpha]^{20}{ }_{\mathrm{D}}=+8.8\left(c 0.54, \mathrm{CHCl}_{3}\right)$; IR (film) $3528,3310,2965,2165 \mathrm{~cm}^{-1} ;{ }^{1} \mathrm{H}$ NMR $\left(300 \mathrm{MHz}, \mathrm{CDCl}_{3}\right) \_3.96(1 \mathrm{H}, \mathrm{dd}, J=1.8,9.9$ $\mathrm{Hz}), 3.61(1 \mathrm{H}, \mathrm{dd}, J=2.4,6.0 \mathrm{~Hz}), 2.73(1 \mathrm{H}, \mathrm{dq}, J=2.4,7.2 \mathrm{~Hz}), 2.55(1 \mathrm{H}, \mathrm{m}), 2.30$ $(1 \mathrm{H}, \mathrm{m}), 2.06(1 \mathrm{H}, \mathrm{d}, J=2.4 \mathrm{~Hz}), 1.30(3 \mathrm{H}, \mathrm{d}, J=6.6 \mathrm{~Hz}), 1.22(3 \mathrm{H}, \mathrm{d}, J=7.2 \mathrm{~Hz}), 0.92$ $(3 \mathrm{H}, \mathrm{d}, J=6.9 \mathrm{~Hz}), 0.91(9 \mathrm{H}, \mathrm{s}), 0.15(9 \mathrm{H}, \mathrm{s}), 0.09(6 \mathrm{H}, \mathrm{d}, J=4.2 \mathrm{~Hz}) ;{ }^{13} \mathrm{C} \mathrm{NMR}_{-}$ $\left(75.5 \mathrm{MHz}, \mathrm{CDCl}_{3}\right)$ 109.52, 97.28, 86.61, 77.25, 73.05, 72.88, 70.07, 39.93, 30.71, $25.92,18.34,17.03,9.36,-0.02,-4.35$.

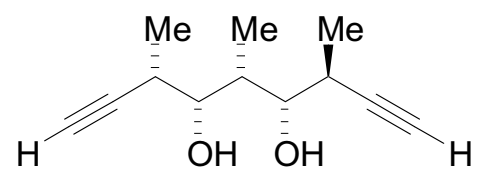

$(3 S, 4 S, 5 S, 6 R, 7 S)-(+)-3,5,7-T r i m e t h y l n o n a n-1,8-d i y n e-$ (1.0M in THF, $12.0 \mathrm{~mL}, 12.0 \mathrm{mmol}$ ). After $3 \mathrm{~h}$ the reaction was judged to be complete 
by TLC and was quenched by the addition of saturated aqueous $\mathrm{NH}_{4} \mathrm{Cl}(20 \mathrm{~mL})$ and EtOAc $(10 \mathrm{~mL})$. The layers were separated, and the aqueous layer was extracted with EtOAc. The combined organic extracts were washed with brine, dried over $\mathrm{MgSO}_{4}$, filtered, and concentrated under reduced pressure. Purification by column chromatography (15\% EtOAc/hexanes) afforded $0.46 \mathrm{~g}(100 \%)$ of a yellow oil. $\mathrm{R}_{\mathrm{f}}=0.26$ (25\% EtOAc/hexanes); $[\alpha]^{20}=+2.1$ (c 4.1, $\mathrm{CHCl}_{3}$ ); IR (film) 3315, 3266, 2976, 2951, $2871 \mathrm{~cm}^{-1}$; ${ }^{1} \mathrm{H}$ NMR $\left(300 \mathrm{MHz}, \mathrm{CDCl}_{3}\right) \_3.65(2 \mathrm{H}, \mathrm{dq}, J=0.9,9.0 \mathrm{~Hz}), 2.68(1 \mathrm{H}, \mathrm{m})$, $2.56(1 \mathrm{H}, \mathrm{m}), 2.30(1 \mathrm{H}, \mathrm{m}), 2.20(1 \mathrm{H}, \mathrm{d}, J=2.4 \mathrm{~Hz}), 2.08(1 \mathrm{H}, \mathrm{d}, J=2.4 \mathrm{~Hz}), 1.32(3 \mathrm{H}$, $\mathrm{d}, J=6.9 \mathrm{~Hz}), 1.18(3 \mathrm{H}, \mathrm{d}, J=6.9 \mathrm{~Hz}), 0.93(3 \mathrm{H}, \mathrm{d}, J=7.2 \mathrm{~Hz}) ;{ }^{13} \mathrm{C} \mathrm{NMR} \_(75.5 \mathrm{MHz} \text {, }$ $\left.\mathrm{CDCl}_{3}\right)$ 86.04, 85.65, 79.79, 79.40, 71.30, 70.19, 35.76, 31.26, 30.18, 17.80, 16.76, 4.26.

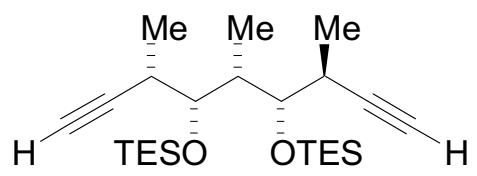

\section{$(3 S, 4 S, 5 S, 6 R, 7 S)-(+)-3,5,7-T r i m e t h y l-4,6-$} bis(triethylsilyloxy)nonan-1,8-diyne (ent-10). To a stirring solution of diol ent-9 $(0.457 \mathrm{~g}, 2.4 \mathrm{mmol})$ in $\mathrm{CH}_{2} \mathrm{Cl}_{2}$ (24 mL) was added 2,6-lutidine $(1.10 \mathrm{~mL}, 9.4 \mathrm{mmol})$. The reaction was cooled to $0{ }^{\circ} \mathrm{C}$, and TESOTf $(1.2 \mathrm{~mL}, 5.2 \mathrm{mmol})$ was subsequently added. After $2 \mathrm{~h}$, the reaction was quenched by addition of $\mathrm{NH}_{4} \mathrm{Cl}(20 \mathrm{~mL})$ and $\mathrm{Et}_{2} \mathrm{O}(10 \mathrm{~mL})$. The layers were separated and the aqueous layer was washed with $\mathrm{Et}_{2} \mathrm{O}$. The combined organic extracts were washed with brine, dried over $\mathrm{MgSO}_{4}$, filtered, and concentrated under reduced pressure. Purification by column chromatography ( $0.5 \%$ EtOAc/hexanes) afforded $0.806 \mathrm{~g}(81 \%)$ of ent-10 as a yellow oil. $\mathrm{R}_{\mathrm{f}}=0.90$ (25\% EtOAc/hexanes); $[\alpha]^{20}{ }_{\mathrm{D}}=+0.8\left(c\right.$ 1.13, $\left.\mathrm{CHCl}_{3}\right)$; IR (film) 3327, 2960, $2890 \mathrm{~cm}^{-1} ;{ }^{1} \mathrm{H}$ NMR $(300 \mathrm{MHz}$, $\left.\mathrm{CDCl}_{3}\right)_{-} 3.77(1 \mathrm{H}, \mathrm{dt}, J=1.2,5.4 \mathrm{~Hz}), 3.57(1 \mathrm{H}, \mathrm{dt}, J=1.2,4.5 \mathrm{~Hz}), 2.70(2 \mathrm{H}, \mathrm{m}), 2.06$ $(3 \mathrm{H}, \mathrm{m}), 1.19(6 \mathrm{H}, \mathrm{qd}, J=1.5,7.2 \mathrm{~Hz}), 0.94-1.03(21 \mathrm{H}, \mathrm{m}), 0.67(12 \mathrm{H}, \mathrm{q}, J=7.5 \mathrm{~Hz})$; ${ }^{13} \mathrm{C} \mathrm{NMR}_{-}\left(75.5 \mathrm{MHz}, \mathrm{CDCl}_{3}\right)$ 87.79, 86.55, 76.16, 75.88, 70.22, 69.69, 40.30, 31.30, $30.17,17.72,15.71,10.91,7.09,5.58$.

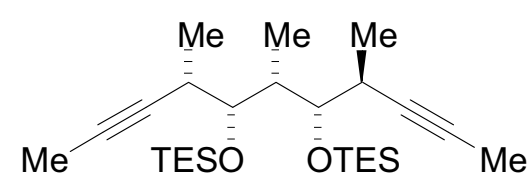

$(3 S, 4 S, 5 S, 6 R, 7 S)-(-)-4,6,8-T r i m e t h y l-5,7-$ bis(triethylsilyloxy)undecan-2,9-diyne (ent-11). To a 
stirring solution of bis-alkyne ent-10 $(0.710 \mathrm{~g}, 1.70 \mathrm{mmol})$ in THF $(17 \mathrm{~mL})$ at $-78{ }^{\circ} \mathrm{C}$ was added BuLi (1.6 M in hexanes, $5.3 \mathrm{~mL}, 8.5 \mathrm{mmol})$. After $1 \mathrm{~h},(\mathrm{MeO})_{2} \mathrm{SO}_{2}(1 \mathrm{~mL}, 10.2$ mmol) was added. After $15 \mathrm{~min}$, the reaction mixture was warmed to $0{ }^{\circ} \mathrm{C}$ for $1 \mathrm{~h}$, and then warmed to room temperature overnight. The reaction was quenched with $\mathrm{NH}_{4} \mathrm{Cl}(20$ $\mathrm{mL})$ and $\mathrm{Et}_{2} \mathrm{O}(10 \mathrm{~mL})$ and the layers were separated. The aqueous layers were extracted with $\mathrm{Et}_{2} \mathrm{O}$ and the combined organic extracts were washed with brine, dried over $\mathrm{MgSO}_{4}$, filtered, and concentrated under reduced pressure. Purification by column chromatography (1\% EtOAc/hexanes) afforded $0.615 \mathrm{~g}(81 \%)$ of ent-11 as a yellow oil. $\mathrm{R}_{\mathrm{f}}=0.98\left(25 \%\right.$ EtOAc/hexanes); $[\alpha]^{20}{ }_{\mathrm{D}}=-4.8$ (c 0.90, $\left.\mathrm{CHCl}_{3}\right)$; IR (film) 2968, 2925, $2881 \mathrm{~cm}^{-1} ;{ }^{1} \mathrm{H}_{\mathrm{NMR}}\left(300 \mathrm{MHz}, \mathrm{CDCl}_{3}\right)\left(300 \mathrm{MHz}, \mathrm{CDCl}_{3}\right) \_3.72(1 \mathrm{H}, \mathrm{dd}, J=4.2,6.9$ $\mathrm{Hz}), 3.54(1 \mathrm{H}, \mathrm{t}, J=4.8 \mathrm{~Hz}), 2.67(1 \mathrm{H}, \mathrm{m}), 2.59(1 \mathrm{H}, \mathrm{m}), 1.93(1 \mathrm{H}, \mathrm{m}), 1.79(6 \mathrm{H}, \mathrm{s})$, 1.13-1.07 (6H, m), 1.00-0.95 (18H, m), 0.94-0.89 (3H, m), 0.67 (12H, dq, $J=2.4,7.8$ $\mathrm{Hz}) ;{ }^{13} \mathrm{C} \mathrm{NMR}_{-}\left(75.5 \mathrm{MHz}, \mathrm{CDCl}_{3}\right)$ 83.29, 82.01, 77.17, 76.32, 76.08, 39.64, 31.80, $29.82,17.72,15.20,10.89,7.11,5.56,5.52,3.72,3.61$.

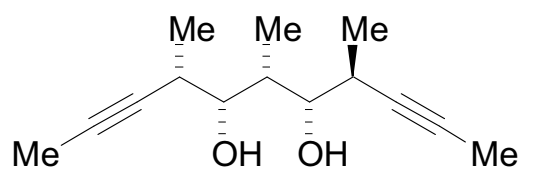
$(4 S, 5 S, 6 S, 7 R, 8 S)-(+)-4,6,8-T r i m e t h y l u n d e c a n-2,9-$ diyne-5,7-diol (ent-12). To a solution of the bis-TES was added TBAF (1.0 M in THF, $9 \mathrm{~mL}, 9 \mathrm{mmol})$. The reaction mixture was stirred for 30 minutes at $0{ }^{\circ} \mathrm{C}$, and then warmed to room temperature for $3 \mathrm{~h}$. The reaction was quenched with saturated aqueous $\mathrm{NH}_{4} \mathrm{Cl}(30 \mathrm{~mL})$ and extracted with ethyl acetate. The extract was washed with brine, dried over $\mathrm{MgSO}_{4}$, filtered, and concentrated under reduced pressure. The crude product was purified by chromatography on silica gel $(15 \%$ EtOAc/hexanes) to afford $0.218 \mathrm{~g}(72 \%)$ of a yellow solid. $\mathrm{R}_{\mathrm{f}}=0.35(25 \%$ EtOAc/hexanes); $[\alpha]^{20}{ }_{\mathrm{D}}=+12.0\left(c 0.81, \mathrm{CHCl}_{3}\right)$; IR (film) $3441,2969,1466 \mathrm{~cm}^{-1} ;{ }^{1} \mathrm{H}$ NMR $\left(300 \mathrm{MHz}, \mathrm{CDCl}_{3}\right)_{-} 3.56(1 \mathrm{H}, \mathrm{dd}, J=1.5,9.3 \mathrm{~Hz}), 3.54(1 \mathrm{H}, \mathrm{dd}, J=2.4,8.7 \mathrm{~Hz})$, $3.01(2 \mathrm{H}$, br s), $2.61(1 \mathrm{H}, \mathrm{dqq}, J=2.4,6.6,6.9 \mathrm{~Hz}), 2.48(1 \mathrm{H}, \mathrm{dqq}, J=2.4,6.6,6.9 \mathrm{~Hz})$, $2.24(1 \mathrm{H}, \mathrm{ddq}, J=2.1,6.9,7.2), 1.82(3 \mathrm{H}, \mathrm{d}, J=2.7 \mathrm{~Hz}), 1.77(3 \mathrm{H}, \mathrm{d}, J=2.4 \mathrm{~Hz}), 1.24$ $(3 \mathrm{H}, \mathrm{d}, J=6.9), 1.12(3 \mathrm{H}, \mathrm{d}, J=6.6 \mathrm{~Hz}), 0.89(3 \mathrm{H}, \mathrm{d}, J=6.9 \mathrm{~Hz}) ;{ }^{13} \mathrm{C} \mathrm{NMR}_{-}(75.5$ 
$\left.\mathrm{MHz}, \mathrm{CDCl}_{3}\right)$ 80.98, 80.10, 80.05, 79.95, 79.03, 77.44, 35.80, 31.40, 30.36, 18.16, $17.23,4.36,3.49$.

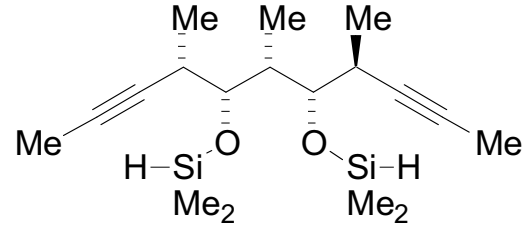

reaction mixture was stirred at room temperature until the solid diol dissolved, and then was heated to $80^{\circ} \mathrm{C}$. After $6 \mathrm{hr}$, the reaction mixture was cooled to room temperature and concentrated via rotary evaporation to remove excess silazane. The crude oil was heated to $60^{\circ} \mathrm{C}$ under high vacuum $(0.03 \mathrm{mmHg})$ overnight. The resulting viscous oil was used in the next step without purification. IR 2977, $2121 \mathrm{~cm}^{-1} ;{ }^{1} \mathrm{H}$ NMR $(300 \mathrm{MHz}$, $\left.\mathrm{CDCl}_{3}\right)_{-} 4.73(1 \mathrm{H}, \mathrm{m}), 4.69(1 \mathrm{H}, \mathrm{m}), 3.62(1 \mathrm{H}, \mathrm{dd}, J=4.5,6.9 \mathrm{~Hz}), 3.43(1 \mathrm{H}, \mathrm{dd}, J=$ $4.5,6.9 \mathrm{~Hz}), 2.71(1 \mathrm{H}, \mathrm{m}), 2.58(1 \mathrm{H}, \mathrm{m}), 2.24(1 \mathrm{H}, \mathrm{dq}, J=4.2,7.2 \mathrm{~Hz}), 1.80(3 \mathrm{H}, \mathrm{d}, J=$ $2.4 \mathrm{~Hz}), 1.77(3 \mathrm{H}, \mathrm{d}, J=2.4 \mathrm{~Hz}), 1.15(3 \mathrm{H}, \mathrm{d}, J=6.9 \mathrm{~Hz}), 1.13(3 \mathrm{H}, \mathrm{d}, J=6.9 \mathrm{~Hz}), 0.92$ $(3 \mathrm{H}, \mathrm{d}, J=6.9 \mathrm{~Hz}), 0.26(3 \mathrm{H}, \mathrm{dd}, J=2.7,3.0 \mathrm{~Hz}), 0.22(3 \mathrm{H}, \mathrm{dd}, J=2.7,5.4 \mathrm{~Hz})$.

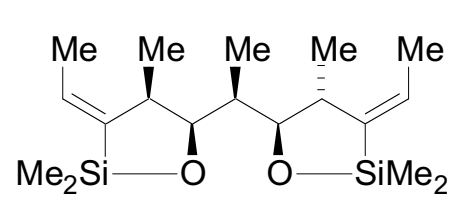

$(4 S, 5 S, 6 S, 7 R, 8 S)$-Bis-Siloxane (ent-14). The residue from the previous step was dissolved in THF $(1.8 \mathrm{~mL})$, and $\mathrm{H}_{2} \mathrm{PtCl}_{6}(0.05 \mathrm{M}$ in THF, $0.05 \mathrm{~mL}, 2.5$ mol $)$ was added. The yellow solution was heated to $75^{\circ} \mathrm{C}$. After $5 \mathrm{~h}$ the hydrosilylation was judged complete by IR analysis ( $\left.\mathrm{Si}-\mathrm{H}, 2121 \mathrm{~cm}^{-1}\right)$. The mixture was diluted with ether and filtered through Celite 521. The mother liquor was concentrated under reduced pressure to afford $0.133 \mathrm{~g}(85 \%)$ of ent-14 as a yellow oil that was used without further purification. IR 2977, $1256 \mathrm{~cm}^{-1} ;{ }^{1} \mathrm{H}$ NMR $\left(300 \mathrm{MHz}, \mathrm{CDCl}_{3}\right){ }_{-} 5.81(2 \mathrm{H}, \mathrm{m}), 4.03(1 \mathrm{H}$, t, $J=2.1 \mathrm{~Hz}), 3.67(2 \mathrm{H}, \mathrm{dd}, J=3.9,9.6 \mathrm{~Hz}), 2.99(1 \mathrm{H}, \mathrm{m}), 2.67(1 \mathrm{H}, \mathrm{q}, J=7.2 \mathrm{~Hz}), 1.76$ $(3 \mathrm{H}, \mathrm{d}, J=6.6 \mathrm{~Hz}), 1.75(3 \mathrm{H}, \mathrm{d}, J=6.3 \mathrm{~Hz}), 1.12(3 \mathrm{H}, \mathrm{d}, J=7.5 \mathrm{~Hz}), 0.91(3 \mathrm{H}, \mathrm{d}, J=6.6$ $\mathrm{Hz}), 0.89(3 \mathrm{H}, \mathrm{d}, J=7.2 \mathrm{~Hz}), 0.24(3 \mathrm{H}, \mathrm{d}, J=3.0 \mathrm{~Hz}), 0.22(3 \mathrm{H}, \mathrm{s}), 0.15(3 \mathrm{H}, \mathrm{s}), 0.07$ $(3 \mathrm{H}, \mathrm{s})$. 


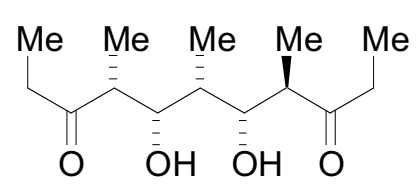

$(4 R, 5 S, 6 R, 7 S, 8 R)-5,7-D i h y d r o x y-4,6,8-$ trimethylundecane-3,9-dione (ent-16). To bis-siloxane ent-14 (0.133 g, $0.40 \mathrm{mmol})$ in $\mathrm{MeOH} / \mathrm{THF}(2: 1,6.8 \mathrm{~mL})$ were added $\mathrm{KF}(0.28$

g, $4.0 \mathrm{mmol})$ and $\mathrm{KHCO}_{3}(0.45 \mathrm{~g}, 4.0 \mathrm{mmol})$ and the reaction mixture was cooled to $0{ }^{\circ} \mathrm{C}$ for 15 minutes. $\mathrm{H}_{2} \mathrm{O}_{2}(7.7 \mathrm{~mL}, 67.5 \mathrm{mmol})$ was added slowly by syringe. After three hours, the reaction was carefully quenched with methanolic $\mathrm{Na}_{2} \mathrm{~S}_{2} \mathrm{O}_{4}(10 \%)$ and diluted with EtOAc. Extraction with EtOAc followed by washing with brine, drying over $\mathrm{MgSO}_{4}$, and concentration under reduced pressure afforded a yellow oil which was carried on without purification. ${ }^{1} \mathrm{H}$ NMR $\left(300 \mathrm{MHz}, \mathrm{CDCl}_{3}\right)_{-} 4.49(1 \mathrm{H}, \mathrm{t}, J=2.4 \mathrm{~Hz})$, $3.45(2 \mathrm{H}, \mathrm{t}, J=3.0 \mathrm{~Hz}), 2.72(1 \mathrm{H}, \mathrm{m}), 2.66(1 \mathrm{H}, \mathrm{dq}, J=7.2,17.7 \mathrm{~Hz}), 2.42(1 \mathrm{H}, \mathrm{dq}, J=$ $7.2,17.7 \mathrm{~Hz}), 2.16(1 \mathrm{H}, \mathrm{dq}, J=7.5 .14 .1 \mathrm{~Hz}), 2.11(1 \mathrm{H}, \mathrm{qd}, J=3.3,7.5 \mathrm{~Hz}), 1.76(1 \mathrm{H}$, m), $1.70(1 \mathrm{H}, \mathrm{dq}, J=7.5,14.1 \mathrm{~Hz}), 1.32(3 \mathrm{H}, \mathrm{d}, J=7.5 \mathrm{~Hz}), 1.07(3 \mathrm{H}, \mathrm{t}, J=7.2 \mathrm{~Hz})$, $1.05(3 \mathrm{H}, \mathrm{d}, J=7.2 \mathrm{~Hz}), 0.98(3 \mathrm{H}, \mathrm{d}, J=6.9 \mathrm{~Hz}), 0.91(3 \mathrm{H}, \mathrm{t}, J=7.8 \mathrm{~Hz})$.

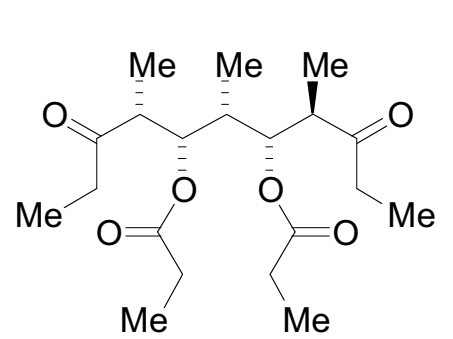

$(4 R, 5 S, 6 R, 7 S, 8 R)$-(-)-bis-Propionic Ester of 5,7Dihydroxy-4,6,8-trimethyl-undecane-3,9-dione (ent-17). To a solution of $\beta$-hydroxy diketone ent-16 from the previous step and propionic acid $(0.17 \mathrm{~mL}, 2.3 \mathrm{mmol})$ in $\mathrm{CH}_{2} \mathrm{Cl}_{2}(4.5$ $\mathrm{mL})$ at $0{ }^{\circ} \mathrm{C}$ was added DMAP $(0.055 \mathrm{~g}, 0.4 \mathrm{mmol})$ and DCC $(0.464 \mathrm{~g}, 2.25 \mathrm{mmol})$. After $12 \mathrm{~h}$, the reaction mixture was filtered through a plug of Celite 521 and concentrated under reduced pressure. Chromatography on silica gel (5\% EtOAc/hexanes) afforded $0.063 \mathrm{~g}$ ( $43 \%$ for 2 steps) of diketo diester ent-17. $\mathrm{R}_{\mathrm{f}}=0.58$ $\left(25 \%\right.$ EtOAc/hexanes); $[\alpha]^{20}{ }_{\mathrm{D}}=-67.8\left(c 0.55, \mathrm{CHCl}_{3}\right)$; IR (film) 2986, 2950, $1736 \mathrm{~cm}^{-1}$; ${ }^{1} \mathrm{H}$ NMR $\left(500 \mathrm{MHz}, \mathrm{CDCl}_{3}\right)_{-} 5.17(1 \mathrm{H}, \mathrm{dd}, J=2.5,9.5 \mathrm{~Hz}), 5.14(1 \mathrm{H}, \mathrm{dd}, J=3.5,9.0$ $\mathrm{Hz}), 3.30(1 \mathrm{H}, \mathrm{dq}, J=3.0,7.0 \mathrm{~Hz}), 2.99(1 \mathrm{H}, \mathrm{m}), 2.25-2.64(8 \mathrm{H}, \mathrm{m}), 2.07(1 \mathrm{H}, \mathrm{m}), 1.02-$ $1.15(15 \mathrm{H}, \mathrm{m}), 0.99(3 \mathrm{H}, \mathrm{t}, J=7.5 \mathrm{~Hz}), 0.91(3 \mathrm{H}, \mathrm{d}, J=6.5 \mathrm{~Hz}) ;{ }^{13} \mathrm{C} \mathrm{NMR} \_(125 \mathrm{MHz} \text {, }$ $\left.\mathrm{CDCl}_{3}\right) 212.01,73.65,73.10,47.56,46.31,35.74,35.45,34.26,27.59,27.46,13.84$, $9.55,9.26,9.11,7.70,7.54$. 


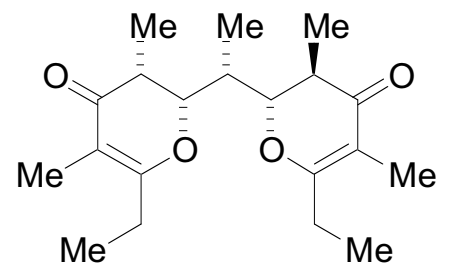

(+)-Membrenone C (ent-18). To diester diketone ent-17 (25 $\mathrm{mg}, 0.06 \mathrm{mmol})$ in $\mathrm{CH}_{2} \mathrm{Cl}_{2}(5.4 \mathrm{~mL})$ at $-78^{\circ} \mathrm{C}$ was added Hunig's base $(0.15 \mathrm{~mL}, 0.86 \mathrm{mmol})$. After $15 \mathrm{~min}$ at $-78^{\circ} \mathrm{C}$, $\mathrm{TiCl}_{4}\left(1.0 \mathrm{M}\right.$ in $\left.\mathrm{CH}_{2} \mathrm{Cl}_{2}, 0.54 \mathrm{~mL}, 0.54 \mathrm{mmol}\right)$ was added

over $1 \mathrm{~h}$. After being stirred for an additional $45 \mathrm{~min}$ at $-78^{\circ} \mathrm{C}$, the reaction mixture was warmed to $-5^{\circ} \mathrm{C}$ for $24 \mathrm{~h}$. The reaction was quenched with $\mathrm{NH}_{4} \mathrm{Cl}$ and extracted with $\mathrm{Et}_{2} \mathrm{O}$. The extract was washed with brine, dried over $\mathrm{MgSO}_{4}$, filtered, and concentrated under reduced pressure. The crude product was purified by chromatography on silica gel $\left(10 \% \mathrm{Et}_{2} \mathrm{O} / \mathrm{CH}_{2} \mathrm{Cl}_{2}\right)$ to afford $10.5 \mathrm{mg}(47 \%)$ of a yellow solid. $\mathrm{R}_{\mathrm{f}}=0.30(10 \%$ $\left.\mathrm{Et}_{2} \mathrm{O} / \mathrm{CH}_{2} \mathrm{Cl}_{2}\right) ;[\alpha]^{20}{ }_{\mathrm{D}}=+23.5$ (c 0.63, $\mathrm{CHCl}_{3}$ ); IR (film) 2986, 2934, 2882, 1675, 1615 $\mathrm{cm}^{-1}$; ${ }^{1} \mathrm{H}$ NMR $\left(500 \mathrm{MHz}, \mathrm{CDCl}_{3}\right) \_4.25(1 \mathrm{H}, \mathrm{dd}, J=2.5,10.0 \mathrm{~Hz}), 3.89(1 \mathrm{H}, \mathrm{dd}, J=$ 2.0, 13.5 Hz), $2.51(1 \mathrm{H}, \mathrm{m}), 2.22-2.46(5 \mathrm{H}, \mathrm{m}), 2.21(1 \mathrm{H}, \mathrm{m}), 1.74(3 \mathrm{H}, \mathrm{s}), 1.71(3 \mathrm{H}, \mathrm{s})$, $1.19(3 \mathrm{H}, \mathrm{d}, J=7.0 \mathrm{~Hz}), 1.18(3 \mathrm{H}, \mathrm{t}, J=8.0 \mathrm{~Hz}), 1.10(3 \mathrm{H}, \mathrm{d}, J=7.0 \mathrm{~Hz}), 1.07(3 \mathrm{H}, \mathrm{t}, J$ $=7.5 \mathrm{~Hz}), 1.02(3 \mathrm{H}, \mathrm{d}, J=7.5 \mathrm{~Hz}) ;{ }^{13} \mathrm{C}$ NMR _ $\left(125 \mathrm{MHz}, \mathrm{CDCl}_{3}\right) 197.10,194.56$, $173.71,172.48,108.66,107.71,81.69,80.94,40.44,39.92$, 34.67, 25.42, 10.91, 10.81, 9.78, 9.32, 9.25, 9.10.

\section{References}

1. Marshall, J. A.; Yanik, M. M. Org. Lett. 2000, 2, 2173.

2. Perkins, M. V.; Sampson, R. A. Org. Lett. 2001, 3, 123. 